\title{
Levothyroxine Dosing After Thyroidectomy Should Be Determined by Both BMI and Body Weight
}

\section{Elizabeth N. Pearce}

Section of Endocrinology, Diabetes, and Nutrition; Department of Medicine, Boston Medical Center, Boston University School of Medicine, Boston, Massachusetts, U.S.A.

Review of: Papoian V, Ylli D, Felger EA, Wartofsky L, Rosen JE 2019 Evaluation of thyroid hormone replacement dosing in overweight and obese patients after a thyroidectomy. Thyroid. Epub 2019 Oct 1. PMID: 31573413.

\section{SUMMARY}

\section{Background}

In patients without thyroid cancer, levothyroxine treatment is frequently initiated at $1.6 \mu \mathrm{g} / \mathrm{kg}$ of body weight following total thyroidectomy. Prior studies have suggested that although obese individuals require higher absolute levothyroxine doses than do individuals who are normal weight, their required doses are lower relative to body weight (1-3). However, there is currently no simple algorithm to guide levothyroxine dosing in obese patients.

\section{Methods}

This was a retrospective analysis of consecutive patients who underwent total thyroidectomy for benign thyroid disease at a single tertiary referral center between 2012 and 2015 (4). Pregnant and lactating women were excluded. Demographics, surgical indication, perioperative and postoperative laboratory results, and surgical pathology were abstracted from the medical record. Time to euthyroidism was the time to the first normal TSH value after surgery. Postoperative levothyroxine dosing was considered to be stable when two consecutive TSH values on the same dose were in the range of 0.45 to $4.50 \mathrm{mIU} / \mathrm{L}$. The preoperative weight of each patient was considered the actual weight (AW). Ideal body weight (IBW) for height was calculated using the Devine formula (5), and lean body weight was calculated based on the LBW 2005 formula
(6). Adjusted body weight was calculated as IBW + 0.4(AW - IBW).

Two-sample t-tests or ANOVA were used to assess relationships between continuous variables, and the chi-square test was used for categorical variables. Linear regression models were used to assess relationships of levothyroxine dosing to measures of body weight.

\section{Results}

A total of 255 patients underwent total thyroidectomy for benign thyroid disease during the study period. Of these, the 114 who had follow-up data and who had become euthyroid while taking levothyroxine were included in analyses. The mean age of the patients was 55 years, and $84 \%$ of them were women. Fifty-two percent had undergone surgery for symptomatic goiter, $45 \%$ for suspicious nodules, and $3 \%$ for Graves' disease. Nearly half (48\%) of patients were obese (BMI $<25$ in 23\%, 25-29 in $29 \%, 30-34$ in 20\%, $35-39$ in $17 \%$, and $>40$ in $11 \%$ ).

Subjects required a mean of 50 weeks to achieve stable euthyroidism postoperatively, and there were no differences in BMI category. In regression models using LBW, adjusted body weight, or body-surface area to predict levothyroxine dosing, there was less variability across calculated coefficients by BMI 

Should Be Determined by Both BMI and Body Weight

category than for models using actual weight. In using actual weights, the required levothyroxine doses differed by $\mathrm{BMI}$ category $(\mathrm{P}<0.01)$ and were: $1.76 \mu \mathrm{g} /$ $\mathrm{kg}$ for those with $\mathrm{BMI}<25,1.47$ for those with $\mathrm{BMI}$ 25-29, 1.42 for those with BMI 30-34, 1.27 for those with $\mathrm{BMI} 35-39$, and 1.28 for those with $\mathrm{BMI}>40$.

\section{Conclusions}

Using actual weight alone to dose levothyroxine after thyroidectomy results in overdosing among overweight and obese patients. It is preferable to consider both actual weight and $\mathrm{BMI}$ in determining the initial levothyroxine dose in this population.

\section{COMMENTARY}

Given that $40 \%$ of U.S. adults were categorized as obese in 2015-2016 and an additional 32\% were categorized as overweight (7), this study addresses a clinically important question. Systematically overdosing obese or overweight patients with levothyroxine after thyroidectomy will lead to the potential for prolonged thyrotoxicosis with concomitant cardiac and bone risks, as well as the need for increased lab testing in order to titrate levothyroxine dosing. Limitations of the study include its retrospective nature and relatively small sample size, as well as the fact that investigators were not able to ascertain patients' compliance with the levothyroxine regimen.
The actual weight-based dosing by BMI category provided in this study seems to be a reasonable and easy-to-implement approach to choosing levothyroxine doses after thyroidectomy. I have incorporated these data in my own practice by making a smartphrase for my electronic medical record which includes the weight-based levothyroxine doses for the different BMI categories. Given that BMI and actual weight are typically both found in electronic medical record, this is a simpler approach than calculating adjusted weights, LBW, or body surface for obese patients. Prospective studies will be needed to determine whether this dosing approach shortens the time to achieve stable euthyroidism.

\section{References}

1. Devdhar M, Drooger R, Pehlivanova M, Singh G, Jonklaas J 2011 Levothyroxine replacement doses are affected by gender and weight, but not age. Thyroid 21:821-827.

2. Glymph K, Gosmanov AR 2016 Levothyroxine replacement in obese hypothyroid females after total thyroidectomy. Endocr Pract 22:22-29.

3. Elfenbein DM, Schaefer S, Shumway C, Chen H, Sippel RS, Schneider DF 2016 Prospective intervention of a novel levothyroxine dosing protocol based on body mass index after thyroidectomy. J Am Coll Surg 222:83-88.
4. Papoian V, Ylli D, Felger EA, Wartofsky L, Rosen JE 2019 Evaluation of thyroid hormone replacement dosing in overweight and obese patients after a thyroidectomy. Thyroid. Epub 2019 Oct 1.

5. Devine B 1974 Gentamicin therapy. Drug Intell Clin Pharm 8:650-655.

6. Janmahasatian S, Duffull SB, Ash S, Ward LC, Byrne NM, Green B 2005. Quantification of lean bodyweight. Clin Pharmacokinet 44:1051-1065.

7. National Center for Health Statistics. Obesity and overweight. https://www.cdc.gov/nchs/fastats/ obesity-overweight.htm. 